\title{
Seite der Therapie - Page de thérapeutique
}

Davidson, Ch. S.: Diet in the treatment of liver disease. Amer. J. Med. 25: 690-697 (nov. 1958). Le role d'une alimentation défectueuse est essentiel dans le déterminisme des affections hépatiques chroniques: il comporte aussi bien des carences dans $\Gamma$ apport alimentaire que dans Tabsorption et dans le métabolisme intermédiaire.

Dans Vhêpatíte à virus, une ration alimentaire riche - $3000 \mathrm{Cal}$., dont $150 \mathrm{~g}$. de protides et de lipides - accélère la guérison complete, mais ne suffit pas à prevenir revolution vers la chronicité.

Dans la cirrhos $\beta$ alcoolíque, les carences sont multiples et complexes et exigent des apports vitaminiques (médicamenteux) à haute dose, en plus de 1'alimentation essentiellement glucidoprotidique.

Dans la cirrhose chroníque, il existe toujours une grave dénutrition (plus qu'une malnutrition), qui sera corrigée par une ration normale, additionnée d'extraits pancréatiques. En presence d'ascite ou d'cedèmes, la désodation s'impose - au maximum $500 \mathrm{mg}$. Na par jour, parfois 200 mg. seulement - ce qui complique singulièrement Гingestion d'une ration caloriquement suffisante.

Dans le coma hépatíque, on constate la presence de diverses substances toxiques, d'origine intestinale vraisemblable. Les protéines alimentaires ne sont habituelle-ment pas responsables de cette auto-intoxication, mais doivent être réduites à moins de $50 \mathrm{~g}$. par jour, tandis que les antibiotiques freineront les processus micro-biens intestinaux nocifs.

Les interventions chírurgícales importantes sont généralement mal supportées par les cirrhotiques, et la preparation nutritionnelle spécialement importante dans ces cas. Pettinarí, V.: Moderni indirrizzi nel trattamento chirurgico del caiicro dell'esofago toracico. (Directives modernes dn traitement chirurgical du cancer de Pcesophage thoracique.) Chir. ital. 9 (1957).

L'expérience de 118 cas appuyée sur une revision clinique de la littérature per-met à Tauteur de préciser les regies qu'il suit dans le traitement chirurgical du cancer de $\Gamma$ oesophage thoracique. Le cancer de l'æsophage inférieur doit toujours être enlevé lorsque les conditions 